\title{
On the solutions for box differential equations under generalized Hukuhara derivative
}

\section{Le Thanh Quang*}

\section{"Correspondence:}

lethanhquang.edu.vn@gmail.com

Faculty of Mathematics and

Computer Science, University of

Science, Hochiminh City, Vietnam

\begin{abstract}
In this paper, the box differential equations under generalized Hukuhara derivatives are considered. We study the existence and uniqueness results for box integral equations employing the method of upper and lower solutions. Moreover, the approach is followed to prove the existence of solutions for the initial value problem of box differential equations. The method is illustrated by some examples.
\end{abstract}

Keywords: box differential equations; box integral equations; generalized Hukuhara derivative

\section{Introduction}

The set-valued differential and integral equations are an important part of the theory of set-valued analysis, and they have the high value for control theory and its application. They were first studied in 1969 by De Blasi and Iervolino [1]. Recently, set-valued differential equations have been studied by many scientists due to their applications in many areas. For many results in the theory of set-valued differential and integral equations, the readers are referred to the following books and papers [2-10] and references therein.

In many real-word problems, it is desirable to transforms the behavior of a special phenomenon into a deterministic initial value problem of linear systems of differential equations, namely,

$$
X^{\prime}(t)=A X(t)+F(t), \quad X(0)=X_{0},
$$

where $A=\left(a_{i j}\right) \in M^{n}(\mathbb{R}), X=\left(x_{1}, x_{2}, \ldots, x_{n}\right), X_{0}=\left(x_{10}, x_{20}, \ldots, x_{n 0}\right) \in \mathbb{R}^{n}, t \in[0, T]$, and $F=\left(f_{1}, f_{2}, \ldots, f_{n}\right):[0, T] \rightarrow \mathbb{R}^{n}$. However, the model is not usually perfect due to the lack of certain information of the initial value $X_{0}$, matrix $A$ or $F$, which must be estimated through measurements. The analysis of measurements errors leads to the study of qualitative behavior of the solutions of (1.1). In such situations, interval-valued differential equations (IDEs) are common tools if the underlying structure is not probabilistic. The intervalvalued analysis and interval-valued differential equations are special cases of set-valued analysis and set-valued differential equations, respectively. In many cases, when modeling real-world phenomena, information about the behavior of a dynamical system is uncertain and one has to consider these uncertainties to gain better understanding of the full

(C) 2014 Quang; licensee Springer. This is an Open Access article distributed under the terms of the Creative Commons Attribution License (http://creativecommons.org/licenses/by/2.0), which permits unrestricted use, distribution, and reproduction in any medium, provided the original work is properly cited. 
models. The interval-valued differential equations can be used to model dynamical systems subjected to uncertainties. The papers of Stefanini and Bede [11, 12], Malinowski $[13,14]$, and An et al. $[15,16]$ are focused on the interval-valued differential equations. These equations can be studied with a framework of the Hukuhara derivative [17]. However, because of this the solutions have increasing length of their values. Furthermore, we notice that this definition of Hukuhara derivative is very restrictive; for instance we can see that if $X(t)=C \cdot x(t)$, where $C$ is an interval-valued constant and $x:[a, b] \rightarrow \mathbb{R}_{+}$is a real-valued function with $x^{\prime}(t)<0$, then $X(t)$ is not differentiable. Recently, to avoid this difficulty, Stefanini and Bede [11] proposed to consider a so-called generalized derivative of interval-valued functions. The interval-valued differential equations with this derivative can have solutions with decreasing length of their values, but the uniqueness is lost. The paper of Stefanini and Bede was the starting point for the topic of interval-valued differential equations (see Malinowski [13, 14], An et al. [15]) and later also for fuzzy differential equations. The connection between the fuzzy analysis and the interval analysis is very well known (Moore and Lodwick [18]). Interval analysis and fuzzy analysis were introduced as an attempt to handle interval uncertainty that appears in many mathematical or computer models of some deterministic real-world phenomena. The main theoretical and practical results in the fields of fuzzy analysis and the interval analysis can be found in several works (Moore [19], Alefeld and Mayer [20], Kolev [21]). Furthermore, some very important extensions of the interval-valued differential equations are the set-valued differential equations $[8,9,22]$, the fuzzy differential equations [23-32], the random fuzzy differential equations [33-37], and fuzzy stochastic differential equations [38-42]; and some methods for solving fuzzy differential equations are presented e.g. in [43-50].

Based on the extensions of (1.1) for the interval cases, in this paper, we study the initial value problem of interval-valued nonlinear systems of differential equations under generalized Hukuhara differentiability, i.e., box-valued differential equations of the form

$$
D_{\mathrm{gH}} X(t)=F(t, X(t)), \quad X(0)=X_{0} \in \mathbb{I}^{n}, \quad t \in[0, T]
$$

where $X=\left(X_{1}, X_{2}, \ldots, X_{n}\right), X_{0}=\left(X_{10}, X_{20}, \ldots, X_{n 0}\right) \in \mathbb{I}^{n}, t \in[0, T]$ and $F=\left(F_{1}, F_{2}, \ldots, F_{n}\right)$ : $[0, T] \times \mathbb{I}^{n} \rightarrow \mathbb{I}^{n}(\mathbb{I}^{n}=\underbrace{\mathbb{I} \times \mathbb{I} \times \cdots \times \mathbb{I}}_{n \text { times }}, \mathbb{I}$ is the space of interval-valued functions). In [11, 13-15, 51-53] the authors studied box-valued differential equations under generalized Hukuhara differentiability for the case $n=1$. The existence and uniqueness of an initial value problem are then obtained under an assumption that the coefficients satisfy a condition with the Lipschitz constant (see [11]). The proof is based on the application of the Banach fixed point theorem. In [14], under the generalized Lipschitz condition, Malinowski obtained the existence and uniqueness of solutions to both kinds of IDEs. In this paper, we study the existence and uniqueness of solutions of two forms of the box integral equations. We employ a the method of upper and lower solutions. The first one leads to the solutions which possess the trajectories with nonincreasing diameter of their values. In the second form the solutions have the trajectories with nondecreasing diameter of their values. Moreover, the approach is applied to prove the existence of solutions for the boxvalued differential equations under generalized Hukuhara derivative (1.2).

This paper is organized as follows: In Section 2, we present some needed concepts about differentiation and integration of box-valued functions. In Section 3, we study the exis- 
tence and uniqueness theorems of solutions for two general forms of box integral equations by the method of upper and lower solutions. We use these results in order to investigate the existence results of solutions for the initial value problem of box differential equations. Finally, we give some examples being simple illustrations of the theory of boxvalued differential equations.

\section{Preliminaries}

Let us denote by $\mathbb{I}$ the set of any nonempty compact intervals of the real line $\mathbb{R}$. The addition and scalar multiplication in $\mathbb{I}$, are defined as usual, i.e. for $A, B \in \mathbb{I}, A=[\underline{A}, \bar{A}]$, $B=[\underline{B}, \bar{B}]$, where $\underline{A} \leq \bar{A}, \underline{B} \leq \bar{B}$, and $\lambda \geq 0$, then we have

$$
A+B=[\underline{A}+\underline{B}, \bar{A}+\bar{B}], \quad \lambda A=[\lambda \underline{A}, \lambda \bar{A}] \quad(-\lambda A=[-\lambda \bar{A},-\lambda \underline{A}]) .
$$

Furthermore, let $A \in \mathbb{I}, \lambda_{1}, \lambda_{2}, \lambda_{3}, \lambda_{4}, \in R$ and $\lambda_{3} \lambda_{4} \geq 0$, then we have $\lambda_{1}\left(\lambda_{2} A\right)=\left(\lambda_{1} \lambda_{2}\right) A$ and $\left(\lambda_{3}+\lambda_{4}\right) A=\lambda_{3} A+\lambda_{4} A$. Let $A, B \in \mathbb{I}$ as above, the Hausdorff metric $H$ in $\mathbb{I}$ is defined as follows:

$$
H[A, B]=\max \{|\underline{A}-\underline{B}|,|\bar{A}-\bar{B}|\} .
$$

We notice that $(\mathbb{I}, H)$ is a complete, separable and locally compact metric space. The magnitude and the length of $A \in \mathbb{I}$ are defined by

$$
H[A,\{0\}]=\|A\|=\max \{|\underline{A}|,|\bar{A}|\}, \quad \operatorname{len}(A)=\bar{A}-\underline{A},
$$

respectively, where $\{0\}$ is the zero element of $\mathbb{I}$, which is regarded as one point.

The generalized Hukuhara difference (or gH-difference) of two intervals $A=[\underline{A}, \bar{A}], B=$ $[\underline{B}, \bar{B}]$ is defined as follows:

$$
A \ominus_{\mathrm{gH}} B=[\underline{A}, \bar{A}] \ominus_{\mathrm{gH}}[\underline{B}, \bar{B}]=[\min \{\underline{A}-\underline{B}, \bar{A}-\bar{B}\}, \max \{\underline{A}-\underline{B}, \bar{A}-\bar{B}\}] .
$$

In the present paper, we introduce the so-called boxes and box-valued functions. Let now $\mathbb{I}^{n}=\mathbb{I} \times \mathbb{I} \times \cdots \times \mathbb{I}$ ( $n$ times) (the Cartesian product) denote the family of axis-aligned boxes (boxes for short) of the $\mathbb{R}^{n}$. An element $A \in \mathbb{I}^{n}$ is of the form $A=\left[\underline{A}_{1}, \bar{A}_{1}\right] \times\left[\underline{A}_{2}, \bar{A}_{2}\right] \times$ $\cdots \times\left[\underline{A}_{n}, \bar{A}_{n}\right]=\prod_{i=1}^{n} A_{i}$, for short, where $A_{i}=\left[\underline{A}_{i}, \bar{A}_{i}\right] \in \mathbb{I}$ are real compact intervals.

If $A=\prod_{i=1}^{n}\left[\underline{A}_{i}, \bar{A}_{i}\right], B=\prod_{i=1}^{n}\left[\underline{B}_{i}, \bar{B}_{i}\right]$ in $\mathbb{I}^{n}$ where $\underline{A}_{i} \leq \bar{A}_{i}, \underline{B}_{i} \leq \bar{B}_{i}$, then the operations on the boxes are defined by

$$
A+B=\prod_{i=1}^{n}\left[\underline{A}_{i}+\underline{B}_{i}, \bar{A}_{i}+\bar{B}_{i}\right] \quad \text { and } \quad \lambda A= \begin{cases}\prod_{i=1}^{n}\left[\lambda \underline{A}_{i}, \lambda \bar{A}_{i}\right], & \text { if } \lambda>0 \\ \{0\}, & \text { if } \lambda=0 \\ \prod_{i=1}^{n}\left[\lambda \bar{A}_{i}, \lambda \underline{A}_{i}\right], & \text { if } \lambda<0 .\end{cases}
$$

The metric structure is given by the Hausdorff distance $H_{B}: \mathbb{I}^{n} \times \mathbb{I}^{n} \rightarrow \mathbb{R}^{+} \cup\{0\}$,

$$
H_{B}[A, B]=\left(\sum_{i=1}^{n} H^{2}\left[A_{i}, B_{i}\right]\right)^{1 / 2}=\left(\sum_{i=1}^{n}\left\{\max \left[\left|\underline{A}_{i}-\underline{B}_{i}\right|,\left|\bar{A}_{i}-\bar{B}_{i}\right|\right]\right\}^{2}\right)^{1 / 2} .
$$


Based on the properties of $(\mathbb{I}, H)$, see $[11,12]$, we deduce that $\left(\mathbb{I}^{n}, H_{B}\right)$ is a complete, separable metric space. For the metric $H_{B}$ the following properties hold:

$$
\begin{aligned}
& H_{B}[A+B, C+D] \leq H_{B}[A, C]+H_{B}[B, D], \\
& H_{B}[\lambda A, \lambda B]=|\lambda| H_{B}[A, B]
\end{aligned}
$$

for every $A, B, C, D \in \mathbb{I}^{n}$, and every $\lambda \in \mathbb{R}$.

The gH-difference of two boxes $A=\prod_{i=1}^{n} A_{i}, B=\prod_{i=1}^{n} B_{i}$ is defined as follows:

$$
A \ominus_{\mathrm{gH}} B= \begin{cases}\prod_{i=1}^{n}\left[\underline{A}_{i}-\underline{B}_{i}, \bar{A}_{i}-\bar{B}_{i}\right], & \text { if } \operatorname{len}\left(A_{i}\right) \geq \operatorname{len}\left(B_{i}\right), \forall i, \\ \prod_{i=1}^{n}\left[\bar{A}_{i}-\bar{B}_{i}, \underline{A}_{i}-\underline{B}_{i}\right], & \text { if } \operatorname{len}\left(A_{i}\right)<\operatorname{len}\left(B_{i}\right), \forall i\end{cases}
$$

If $A, B, C \in \mathbb{I}^{n}$ then it is easy to see that

$$
A \ominus_{\mathrm{gH}} B=C \Leftrightarrow \begin{cases}A=B+C, & \text { if len }\left(A_{i}\right) \geq \operatorname{len}\left(B_{i}\right), \forall i, \\ B=A+(-C), & \text { if } \operatorname{len}\left(A_{i}\right)<\operatorname{len}\left(B_{i}\right), \forall i .\end{cases}
$$

Proposition 2.1 Let $A=\prod_{i=1}^{n} A_{i}, B=\prod_{i=1}^{n} B_{i} \in \mathbb{I}^{n}$. The gH-difference $A \ominus_{\mathrm{gH}} B$ exists if and only if one of the two conditions is satisfied:

(i) $\operatorname{len}\left(A_{i}\right) \geq \operatorname{len}\left(B_{i}\right), i=1,2, \ldots, n$,

or

(ii) $\operatorname{len}\left(A_{i}\right) \leq \operatorname{len}\left(B_{i}\right), i=1,2, \ldots, n$.

Based on the gH-difference and the gH-derivative of interval-valued functions (see [11]) we propose the following.

Definition 2.1 Let $t_{0} \in[a, b]$ and $h$ be such that $t_{0}+h \in[a, b]$, the gH-derivative of a function $X:[a, b] \rightarrow \mathbb{I}^{n}$ at $t_{0}$ is defined as

$$
D_{\mathrm{gH}} X\left(t_{0}\right)=\lim _{h \rightarrow 0} \frac{1}{h}\left[X\left(t_{0}+h\right) \ominus_{\mathrm{gH}} X\left(t_{0}\right)\right]
$$

If $D_{\mathrm{gH}} X\left(t_{0}\right) \in \mathbb{I}^{n}$ satisfying (2.4) exists, we say that $X$ is generalized Hukuhara differentiable (gH-differentiable for short) at $t_{0}$.

Let us remark that the $\mathrm{gH}$-derivative exists at $t_{0}$ if and only if the left and right derivatives at $t_{0}$ exist and they are equal. We say that $X$ is gH-differentiable on $[a, b]$ if $D_{\mathrm{gH}} X(t) \in \mathbb{I}^{n}$ exists at each point $t \in[a, b]$. At the end points of $[a, b]$ we consider only the one sided gH-derivatives.

Definition 2.2 Let $X:[a, b] \rightarrow \mathbb{I}^{n}$ be such that $X(t)=\prod_{i=1}^{n} X_{i}(t)=\prod_{i=1}^{n}\left[\underline{X}_{i}(t), \bar{X}_{i}(t)\right]$ and gH-differentiable at $t_{0} \in[a, b]$. We say that $X$ is $\left((\mathrm{i})\right.$-gH)-differentiable at $t_{0}$ if

$$
D_{\mathrm{gH}} X\left(t_{0}\right)=\prod_{i=1}^{n}\left[\frac{d}{d t} \underline{X}_{i}\left(t_{0}\right), \frac{d}{d t} \bar{X}_{i}\left(t_{0}\right)\right]
$$

and that $X$ is ((ii)-gH)-differentiable at $t_{0}$ if 
(ii)

$$
D_{\mathrm{gH}} X\left(t_{0}\right)=\prod_{i=1}^{n}\left[\frac{d}{d t} \bar{X}_{i}\left(t_{0}\right), \frac{d}{d t} \underline{X}_{i}\left(t_{0}\right)\right]
$$

Definition 2.3 Let $X:[a, b] \rightarrow \mathbb{I}^{n}$ be such that $X(t)=\prod_{i=1}^{n} X_{i}(t)=\prod_{i=1}^{n}\left[\underline{X}_{i}(t), \bar{X}_{i}(t)\right]$ and $\underline{X}_{i}(t), \bar{X}_{i}(t), i \in\{1,2, \ldots, n\}$ are measurable and Lebesgue integrable on $[a, b]$. Then the integral of box-valued function $X$ is defined as usually:

$$
\int_{a}^{b} X(t) d t=\prod_{i=1}^{n}\left[\int_{a}^{b} \underline{X}_{i}(t) d t, \int_{a}^{b} \bar{X}_{i}(t) d t\right]
$$

We say that $X$ is Lebesgue integrable on $[a, b]$. It is easy to show that the box-valued function $X$ is integrable on $[a, b]$ if and only if $X$ is measurable and $H_{B}[X(t),\{0\}]$ is Lebesgue integrable on $[a, b]$.

In this paper, we use the following partial ordering (see [7]).

Definition 2.4 Suppose $A=\prod_{i=1}^{n}\left[\underline{A}_{i}, \bar{A}_{i}\right], B=\prod_{i=1}^{n}\left[\underline{B}_{i}, \bar{B}_{i}\right]$. We say that $A \leq B$ if and only if $\underline{A}_{i} \leq \underline{B}_{i}$ and $\bar{A}_{i} \leq \bar{B}_{i}$, for $i=1, \ldots, n$.

We can also define the interval $[A, B]=\left\{C \in \mathbb{I}^{n}: A \leq C \leq B\right\}$. Moreover, letting $X, Y \in$ $C\left([a, b], \mathbb{I}^{n}\right)$ be two box-valued functions, we say that $X \leq Y$ if $X(t) \leq Y(t)$ for $t \in[a, b]$. We define $[X(t), Y(t)]=\left\{Z \in C\left([a, b], \mathbb{I}^{n}\right): X(t) \leq Z(t) \leq Y(t)\right\}$.

We recall some properties on the partial ordering $\leq$ the space of box-valued functions, which are useful for our procedure.

Proposition 2.2 Let $A, B, C, D \in \mathbb{I}^{n}$ and $c \in \mathbb{R}_{+}$,

(i) $A=B$ if and only if $A \leq B$ and $A \geq B$;

(ii) if $A \leq B$, then $A+C \leq B+C$;

(iii) if $A \leq B$ and $C \leq D$, then $A+C \leq B+D$;

(iv) if $A \leq B$, then $c A \leq c B$.

Lemma 2.1 ([26]) Let $F, G, H \in C\left([0, T], \mathbb{I}^{n}\right)$ and $X_{0} \in \mathbb{I}^{n}$ and also $G \leq H$, then

(i) $\int_{0}^{t} G(s) d s \leq \int_{0}^{t} H(s) d s, t \in[0, T]$;

(ii) $X_{0} \ominus(-1) \int_{0}^{t} G(s) d s \leq X_{0} \ominus(-1) \int_{0}^{t} H(s) d s, t \in[0, T]$;

(iii) if $G(t) \leq F(t) \leq H(t), t \in[0, T]$, then $\int_{0}^{t} F(s) d s$ is well defined on $[0, T]$.

The following theorem is similar to the result proved in [11].

Theorem 2.1 Let $X:[a, b] \rightarrow \mathbb{I}^{n}$ be ((i)-gH)-differentiable or ((ii)-gH)-differentiable on $[a, b]$, and assume that the derivative $D_{\mathrm{gH}} X$ is integrable over $[a, b]$. We have

(a) if $X$ be ((i)-gH)-differentiable on $[a, b]$, then $\int_{a}^{b} D_{\mathrm{gH}} X(t) d t=X(b) \ominus X(a)$;

(b) if $X$ be ((ii)-gH)-differentiable on $[a, b]$, then $\int_{a}^{b} D_{\mathrm{gH}} X(t) d t=(-1)(X(a) \ominus X(b))$.

\section{Box differential equations}

In the sequel, we study the existence and uniqueness theorems of solutions for two general forms of box integral equations by the method of upper and lower solutions. We use these 
results in order to investigate the existence results of solutions for the initial value problem of box differential equations.

We consider two forms of the following box integral equations:

$$
X(t)=X_{0}+\int_{0}^{t} F(s, X(s)) d s, \quad t \in[0, T]
$$

and

$$
Y(t)=Y_{0} \ominus(-1) \int_{0}^{t} F(s, Y(s)) d s, \quad t \in[0, T],
$$

where $F \in C\left([0, T] \times \mathbb{I}^{n}, \mathbb{I}^{n}\right)$ and the integral of $F$ is integral from Definition 2.3.

The solutions of equations (3.1) and (3.2) have no crisp elements and different geometrical properties.

Remark 3.1 If $X:[0, T] \rightarrow \mathbb{I}^{n}$ is a solution to (3.1), then the function $t \mapsto \operatorname{diam}(X(t))$ is nondecreasing, where $\operatorname{diam}(Z)$ denotes the diameter of the box $Z$.

Remark 3.2 If $Y:[0, T] \rightarrow \mathbb{I}^{n}$ is a solution to (3.2), then the function $t \mapsto \operatorname{diam}(Y(t))$ is nonincreasing.

Definition 3.1 Let $X_{\mathrm{U}}, X_{\mathrm{L}}, Y_{\mathrm{U}}, Y_{\mathrm{L}} \in C\left([0, T], \mathbb{I}^{n}\right)$, and $F\left(t, X_{\mathrm{U}}(t)\right), F\left(t, X_{\mathrm{L}}(t)\right), F\left(t, Y_{\mathrm{U}}(t)\right)$, $F\left(t, Y_{\mathrm{L}}(t)\right)$ are integrable on $[0, T]$, we say that

(i) $X_{\mathrm{U}}, X_{\mathrm{L}}$ are upper and lower solutions for (3.1), respectively, if

$$
X_{\mathrm{U}}(t) \geq X_{0}+\int_{0}^{t} F\left(s, X_{\mathrm{U}}(s)\right) d s, \quad t \in[0, T]
$$

and

$$
X_{\mathrm{L}}(t) \leq X_{0}+\int_{0}^{t} F\left(s, X_{\mathrm{L}}(s)\right) d s, \quad t \in[0, T]
$$

(ii) $Y_{\mathrm{U}}, Y_{\mathrm{L}}$ are upper and lower solutions for (3.2), respectively, if

$$
Y_{\mathrm{U}}(t) \geq Y_{0} \ominus(-1) \int_{0}^{t} F\left(s, Y_{\mathrm{U}}(s)\right) d s, \quad t \in[0, T]
$$

and

$$
Y_{\mathrm{L}}(t) \leq Y_{0} \ominus(-1) \int_{0}^{t} F\left(s, Y_{\mathrm{L}}(s)\right) d s, \quad t \in[0, T],
$$

provided the involved Hukuhara differences are well defined.

Definition 3.2 Let $X_{\mathrm{L}}, X_{\mathrm{U}}$ be lower and upper solutions for the box integral equations (3.1), $\left[X_{\mathrm{L}}, X_{\mathrm{U}}\right]=\left\{X \in C\left([a, b], \mathbb{I}^{n}\right): X_{\mathrm{L}} \leq X \leq X_{\mathrm{U}}\right\}$. We say that $X \in\left[X_{\mathrm{L}}, X_{\mathrm{U}}\right]$ is a solution for equation (3.1), if $X$ satisfies equation (3.1). Moreover, the solution $X_{\max } \in\left[X_{\mathrm{L}}, X_{\mathrm{U}}\right]$ is a maximal solution of equation (3.1), if

$$
X_{\max }(t) \geq X(t), \quad t \in[0, T]
$$


for any solution $X \in\left[X_{\mathrm{L}}, X_{\mathrm{U}}\right]$. We define a minimal solution $X_{\min } \in\left[X_{\mathrm{L}}, X_{\mathrm{U}}\right]$ as a function satisfying the reverse inequalities.

Definition 3.3 Let $Y_{\mathrm{L}}, Y_{\mathrm{U}}$ be lower and upper solutions for the set integral equations (3.2), $\left[Y_{\mathrm{L}}, Y_{\mathrm{U}}\right]=\left\{Y \in C\left([a, b], \mathbb{I}^{n}\right): Y_{\mathrm{L}} \leq Y \leq Y_{\mathrm{U}}\right\}$. We say that $Y \in\left[Y_{\mathrm{L}}, Y_{\mathrm{U}}\right]$ is a solution for equation (3.2), if $Y$ satisfies equation (3.2). Moreover, the solution $Y_{\max } \in\left[Y_{\mathrm{L}}, Y_{\mathrm{U}}\right]$ is a maximal solution of equation (3.2), if

$$
Y_{\max }(t) \geq Y(t), \quad t \in[0, T]
$$

for any solution $Y \in\left[Y_{\mathrm{L}}, Y_{\mathrm{U}}\right]$. We define a minimal solution $Y_{\min } \in\left[Y_{\mathrm{L}}, Y_{\mathrm{U}}\right]$ as a function satisfying the reverse inequalities.

\section{Theorem 3.1 Assume that}

(i) $X_{\mathrm{L}}, X_{\mathrm{U}} \in C\left([0, T], \mathbb{I}^{n}\right)$ are coupled upper and lower solutions of the box integral equations (3.1) such that $X_{\mathrm{L}} \leq X_{\mathrm{U}}, t \in[0, T]$;

(ii) $F \in C\left([0, T] \times \mathbb{I}^{n}, \mathbb{I}^{n}\right), F(t, X)$ is nondecreasing in $X \in\left[X_{\mathrm{L}}, X_{\mathrm{U}}\right]$ for all $t \in[0, T]$.

Then there exist maximal and minimal solutions $X_{\max }, X_{\min } \in\left[X_{\mathrm{L}}, X_{\mathrm{U}}\right]$ for equation (3.1).

Proof Let us construct two sequences by

$$
V_{n+1}(t)=X_{0}+\int_{0}^{t} F\left(s, V_{n}(s)\right) d s, \quad V_{0}=X_{\mathrm{L}}, \quad t \in[0, T]
$$

and

$$
W_{n+1}(t)=X_{0}+\int_{0}^{t} F\left(s, W_{n}(s)\right) d s, \quad W_{0}=X_{\mathrm{U}}, \quad t \in[0, T]
$$

We claim that $V_{n}$ and $W_{n}$ are well defined, and

$$
\begin{aligned}
X_{\mathrm{L}}(t) & =V_{0}(t) \leq V_{1}(t) \leq \cdots \leq V_{n}(t) \leq \cdots \leq W_{n}(t) \leq \cdots \\
& \leq W_{1}(t) \leq W_{0}(t)=X_{\mathrm{U}}(t), \quad t \in[0, T] .
\end{aligned}
$$

Indeed, from equation (3.3) we get for $n=0$

$$
V_{1}(t)=X_{0}+\int_{0}^{t} F\left(s, V_{0}(s)\right) d s
$$

According to the integrability of $F, V_{1}$ is well defined. Since $V_{0}=X_{\mathrm{L}}$, by Definition 3.1, we get

$$
V_{0}(t) \leq V_{1}(t), \quad t \in[0, T]
$$

Moreover, $X_{\mathrm{L}}(t) \leq X_{\mathrm{U}}(t), F(t, X)$ is nondecreasing in $X$, we get $V_{1}(t) \leq W_{0}(t)$.

As a result, we obtain

$$
V_{0}(t) \leq V_{1}(t) \leq W_{0}(t), \quad t \in[0, T]
$$


We assume inductively

$$
V_{n-1}(t) \leq V_{n}(t) \leq W_{0}(t), \quad t \in[0, T]
$$

and $V_{k}$ are well defined for $k \leq n$. From equation (3.3), we find

$$
V_{n+1}(t)=X_{0}+\int_{0}^{t} F\left(s, V_{n}(s)\right) d s
$$

and

$$
V_{n}(t)=X_{0}+\int_{0}^{t} F\left(s, V_{n-1}(s)\right) d s
$$

Employing the nondecreasing property of function $F$, along with the relation in equation (3.6), we get

$$
F\left(t, X_{\mathrm{L}}\right) \leq F\left(t, V_{n}\right) \leq F\left(t, X_{\mathrm{U}}\right)
$$

By Lemma 2.1, $V_{n+1}$ is well defined and we get

$$
V_{n}(t) \leq V_{n+1}(t) \leq W_{0}(t), \quad t \in[0, T]
$$

Similarly, we can prove that $V_{0} \leq W_{n+1} \leq W_{n}(t) \leq W_{0}$ and $W_{k}$ are well defined for $k=$ $1,2, \ldots$. Next, we prove $V_{n} \leq W_{n}, \forall n \in \mathbb{N}$. Indeed, $V_{0}=X_{\mathrm{L}} \leq X_{\mathrm{U}}=W_{0}$ comes from the assumption. Now, we suppose inductively

$$
V_{n}(t) \leq W_{n}(t), \quad t \in[0, T]
$$

By equations (3.3), (3.4), and applying the fact that $F$ is nondecreasing, equation (3.7), we deduce that

$$
V_{n+1}(t) \leq W_{n+1}(t), \quad t \in[0, T]
$$

Since the sequence of functions $V_{n}(t)$ is monotone nondecreasing and bounded from above, and the sequence of functions $W_{n}(t)$ is monotone nonincreasing and bounded from below, the pointwise limits exist, and these limits are denoted by $X_{\min }$ and $X_{\max }$ such that they satisfy

$$
\lim _{n \rightarrow \infty} V_{n}(t)=X_{\min }(t), \quad \lim _{n \rightarrow \infty} W_{n}(t)=X_{\max }(t), \quad t \in[0, T] .
$$

Next, taking the limits as $n \rightarrow \infty$ in equations (3.3), (3.4), using continuity and monotone nondecreasing of $F$, we infer that

$$
X_{\min }(t)=X_{0}+\int_{0}^{t} F\left(s, X_{\min }(s)\right) d s, \quad t \in[0, T]
$$


and

$$
X_{\max }(t)=X_{0}+\int_{0}^{t} F\left(s, X_{\max }(s)\right) d s, \quad t \in[0, T] .
$$

Moreover, by equation (3.5), we get

$$
V_{0} \leq X_{\min } \leq X_{\max } \leq W_{0}, \quad t \in[0, T]
$$

Now, we claim that $X_{\min }, X_{\max }$ are minimal and maximal solutions of equation (3.1), that is, if $X(t)$ is any solution of equation (3.1) such that

$$
V_{0} \leq X(t) \leq W_{0}, \quad t \in[0, T]
$$

then

$$
V_{0} \leq X_{\min } \leq X(t) \leq X_{\max } \leq W_{0}, \quad t \in[0, T]
$$

Suppose that for some $n$,

$$
V_{n} \leq X(t) \leq W_{n}, \quad t \in[0, T]
$$

By using the monotone nondecreasing property of $F$ and Lemma 2.1, we get

$$
V_{n+1}(t)=X_{0}+\int_{0}^{t} F\left(s, V_{n}(s)\right) d s \leq X_{0}+\int_{0}^{t} F(s, X(t)(s)) d s=X(t), \quad t \in[0, T]
$$

Similarly $W_{n+1} \geq X(t), t \in[0, T]$. Hence, by induction, equation (3.8) is true for all $n \in \mathbb{N}$. Now, taking the limit as $n \rightarrow \infty$, we get equation (3.9). The proof is complete.

Corollary 3.1 If, in addition to the assumptions of Theorem 3.1, F satisfies, whenever $Y \geq$ $Z ; Y, Z \in \mathbb{I}^{n}$,

$$
F(t, Y)+L Z \leq F(t, Z)+L Y, \quad L>0,
$$

then $X_{\min }=X=X_{\max }$ is the unique solution of equation (3.1).

Proof We have $X_{\min }=\prod_{i=1}^{n}\left[\underline{X}_{i, \min }, \bar{X}_{i, \min }\right], X_{\max }=\prod_{i=1}^{n}\left[\underline{X}_{i, \max }, \bar{X}_{i, \max }\right]$.

Since $X_{\min } \leq X_{\max }$, by Definition 2.3 , we get $X_{\max }=X_{\min }+R$, where $R=\prod_{i=1}^{n}\left[\underline{R}_{i}, \bar{R}_{i}\right]$; $\underline{R}_{i}, \bar{R}_{i} \geq 0$, for $i=1, \ldots, n$. By assumption (3.10), we have

$$
\begin{aligned}
\prod_{i=1}^{n} & {\left[\underline{X}_{i, \min }(t), \bar{X}_{i, \min }(t)\right]+\prod_{i=1}^{n}\left[\underline{R}_{i}(t), \bar{R}_{i}(t)\right] } \\
& =\prod_{i=1}^{n}\left[\underline{X}_{i, \max }(t), \bar{X}_{i, \max }(t)\right] \\
& =\prod_{i=1}^{n}\left[\underline{X}_{i 0}, \bar{X}_{i 0}\right]+\prod_{i=1}^{n}\left[\int_{0}^{t} \underline{F}_{i}\left(s, X_{i, \max }(s)\right) d s, \int_{0}^{t} \bar{F}_{i}\left(s, X_{i, \max }(s)\right) d s\right]
\end{aligned}
$$




$$
\begin{aligned}
\leq & \prod_{i=1}^{n}\left[\underline{X}_{i 0}, \bar{X}_{i 0}\right]+\prod_{i=1}^{n}\left[\int_{0}^{t} \underline{F}_{i}\left(s, X_{i, \min }(s)\right) d s, \int_{0}^{t} \bar{F}_{i}\left(s, X_{i, \min }(s)\right) d s\right] \\
& +L \prod_{i=1}^{n}\left[\int_{0}^{t} \underline{R}_{i}(s) d s, \int_{0}^{t} \bar{R}_{i}(s) d s\right] \\
= & \prod_{i=1}^{n}\left[\underline{X}_{i, \min }, \bar{X}_{i, \min }\right]+L \prod_{i=1}^{n}\left[\int_{0}^{t} \underline{R}_{i}(s) d s, \int_{0}^{t} \bar{R}_{i}(s) d s\right]
\end{aligned}
$$

and hence

$$
\underline{R}_{i}(t) \leq L \int_{t_{0}}^{t} \underline{R}_{i}(s) d s, \quad \bar{R}_{i}(t) \leq L \int_{t_{0}}^{t} \bar{R}_{i}(s) d s, \quad i=1, \ldots, n .
$$

The Gronwall inequality implies that $\underline{R}_{i}(t) \leq 0, \bar{R}_{i}(t) \leq 0$ and as a result, $\underline{R}_{i}(t)=0, \bar{R}_{i}(t)=0$ for $i=1, \ldots, n$. Therefore, $X_{\min }=X=X_{\max }$ is the unique solution.

\section{Theorem 3.2 Assume that}

(i) $Y_{\mathrm{U}}, Y_{\mathrm{L}} \in C\left([0, T], \mathbb{I}^{n}\right)$ are coupled upper and lower solutions of the box integral equations (3.2) such that $Y_{\mathrm{L}} \leq Y_{\mathrm{U}}, t \in[0, T]$;

(ii) $F \in C\left([0, T] \times \mathbb{I}^{n}, \mathbb{I}^{n}\right), F(t, Y)$ is nondecreasing in $Y \in\left[Y_{\mathrm{L}}, Y_{\mathrm{U}}\right]$ for all $t \in[0, T]$;

(iii) the sequences

$$
\begin{aligned}
& V_{0}=Y_{\mathrm{L}}, \quad V_{n+1}(t)=X_{0} \ominus(-1) \int_{0}^{t} F\left(s, V_{n}(s)\right) d s, \\
& W_{0}=Y_{\mathrm{U}}, \quad W_{n+1}(t)=X_{0} \ominus(-1) \int_{0}^{t} F\left(s, W_{n}(s)\right) d s
\end{aligned}
$$

for all $t \in[0, T]$ are well defined.

Then there exist maximal and minimal solutions $Y_{\max }, Y_{\min } \in\left[Y_{\mathrm{L}}, Y_{\mathrm{U}}\right]$ for equation (3.2).

Corollary 3.2 If, in addition to the assumptions of Theorem 3.2, F satisfies, whenever $Y \geq$ $Z, Y, Z \in \mathbb{I}^{n}$,

$$
F(t, Y) \leq F(t, Z)+L(Y \ominus Z), \quad L>0,
$$

then $Y_{\min }=Y=Y_{\max }$ is the unique solution of equation (3.2).

Proof The proof is similar Theorem 3.1 and Corollary 3.1 by employing Lemma 2.1.

In the sequel, we study the box-valued differential equations using the concept of generalized Hukuhara differentiability. In this setting, we prove the existence of two solutions, each one corresponding to a different type of differentiability.

We consider the following box-valued differential equation (BDE) with the initial condition:

$$
D_{\mathrm{gH}} X(t)=F(t, X(t)), \quad X(0)=X_{0} \in \mathbb{I}^{n}, \quad t \in[0, T],
$$

where $F:[0, T] \times \mathbb{I}^{n} \rightarrow \mathbb{I}^{n}$ is continuous box-valued function and $X_{0} \in \mathbb{I}^{n}$ is a nontrivial box. In this paper, we consider only ((i)-gH)-differentiable type and ((ii)-gH)-differentiable 
type solutions, i.e. such that there are no switching points in $[0, T]$. Let us remark here that the equation $D_{\mathrm{gH}} X(t)=F(t, X(t))$ is equivalent to $D_{\mathrm{gH}} X(t) \ominus_{\mathrm{gH}} F(t, X(t))=\{0\}$ and also equivalent to $F(t, X(t)) \ominus_{\mathrm{gH}} D_{\mathrm{gH}} X(t)=\{0\}$, but it is not equivalent to $D_{\mathrm{gH}} X(t)-F(t, X(t))=$ $\{0\}$ (here the ' - ' is in the usual interval arithmetic sense).

The following lemma is similar to the result proved in [11].

Lemma 3.1 The box-valued differential equation (3.11) is equivalent to the following integral equation:

$$
X(t) \ominus_{\mathrm{gH}} X(0)=\int_{0}^{t} F(s, X(s)) d s, \quad t \in[0, T] .
$$

Two cases of existence of the gH-difference imply that the integral equation in Lemma 3.1 is actually a unified formulation for one the integral equations

$$
X(t) \ominus X(0)=\int_{0}^{t} F(s, X(s)) d s \quad \text { and } \quad X(0) \ominus X(t)=(-1) \int_{0}^{t} F(s, X(s)) d s,
$$

with $\ominus$ being the classical Hukuhara difference. Now, let $X$ and $Y$ be solutions of equation (3.11) in ((i)-gH)-differentiable type and ((ii)-gH)-differentiable type, respectively, then by using Lemma 3.1 and Theorem 2.1, we have

$$
\begin{aligned}
& X(t)=X_{0}+\int_{0}^{t} F(s, X(s)) d s, \\
& Y(t)=X_{0} \ominus(-1) \int_{0}^{t} F(s, Y(s)) d s .
\end{aligned}
$$

Definition 3.4 Let $X: I \rightarrow \mathbb{I}^{n}$ be a box-valued function which is ((i)-gH)-differentiable. If $X$ and its derivative satisfy problem (3.11), we say that $X$ is a (i)-solution of problem (3.11).

Definition 3.5 Let $Y: I \rightarrow \mathbb{I}^{n}$ be a box-valued function which is ((ii)-gH)-differentiable. If $Y$ and its derivative satisfy problem (3.11), we say that $Y$ is a (ii)-solution of problem (3.11).

In [14] Malinowski proved the existence and uniqueness of solutions to interval-valued differential equations by using generalized Lipschitz conditions. In [11] authors proved the existence and uniqueness of solutions to interval-valued differential equations by using the Banach fixed point theorem. In the sequel, we study the existence and uniqueness results for box integral and differential equations employing the method of upper and lower solutions.

\section{Theorem 3.3 Assume that}

(i) $X_{\mathrm{U}}, X_{\mathrm{L}} \in C\left([0, T], \mathbb{I}^{n}\right)$ are coupled upper and lower solutions of the box integral equations (3.13) such that $X_{\mathrm{L}}(t) \leq X_{\mathrm{U}}(t), t \in[0, T]$;

(ii) $F \in C\left([0, T] \times \mathbb{I}^{n}, \mathbb{I}^{n}\right), F(t, X)$ is nondecreasing in $X \in\left[X_{\mathrm{L}}, X_{\mathrm{U}}\right]$ for all $t \in[0, T]$.

Then there exists at least one solution $X \in\left[X_{\mathrm{L}}, X_{\mathrm{U}}\right]$ for equation (3.11), which is ((i)-gH)differentiable.

Theorem 3.4 Assume that

(i) $Y_{\mathrm{U}}, Y_{\mathrm{L}} \in C\left([0, T], \mathbb{I}^{n}\right)$ are coupled upper and lower solutions of the box integral equations (3.14) such that $Y_{\mathrm{L}}(t) \leq Y_{\mathrm{U}}(t), t \in[0, T]$; 
(ii) $F \in C\left([0, T] \times \mathbb{I}^{n}, \mathbb{I}^{n}\right), F(t, Y)$ is nondecreasing in $Y \in\left[Y_{\mathrm{L}}, Y_{\mathrm{U}}\right]$ for all $t \in[0, T]$;

(iii) the sequences

$$
\begin{aligned}
& V_{0}=Y_{\mathrm{L}}, \quad V_{n+1}(t)=Y_{0} \ominus(-1) \int_{t_{0}}^{t} F\left(s, V_{n}(s)\right) d s, \\
& W_{0}=Y_{\mathrm{U}}, \quad W_{n+1}(t)=Y_{0} \ominus(-1) \int_{t_{0}}^{t} F\left(s, W_{n}(s)\right) d s
\end{aligned}
$$

for all $t \in[0, T]$ are well defined.

Then there exists at least one solution $Y \in\left[Y_{\mathrm{L}}, Y_{\mathrm{U}}\right]$ for equation (3.11), which is ((ii)-gH)differentiable.

Proof Since all the conditions of Theorem 3.2 are fulfilled, there exists at least a solution, say $X$, for the integral (3.13). We wish to show that $X$ is a solution for equation (3.11).

For small $h>0$, we have

$$
\begin{aligned}
X(t+h)+(-1) \int_{t}^{t+h} F(s, X(s)) d s \\
=\int_{0}^{t+h} F(s, X(s)) d s+(-1) \int_{t}^{t+h} F(s, X(s)) d s \\
=\int_{0}^{t} F(s, X(s)) d s+\int_{t}^{t+h} F(s, X(s)) d s+(-1) \int_{t}^{t+h} F(s, X(s)) d s \\
=\int_{0}^{t} F(s, X(s)) d s=X(t) .
\end{aligned}
$$

Hence, we obtain

$$
X(t) \ominus X(t+h)=(-1) \int_{t}^{t+h} F(s, X(s)) d s .
$$

Moreover, we observe that

$$
\begin{aligned}
H_{B}\left[\frac{1}{h} \int_{t}^{t+h} F(s, X(s)) d s, F(t, X(t))\right] & =H_{B}\left[\frac{1}{h} \int_{t}^{t+h} F(s, X(s)) d s, \frac{1}{h} \int_{t}^{t+h} F(t, X(t))\right] \\
& \leq \frac{1}{h} \int_{t}^{t+h} H_{B}[F(s, X(s)), F(t, X(t))] d s \\
& \leq \sup _{|s-t| \leq h} H_{B}[F(s, X(s)), F(t, X(t))]
\end{aligned}
$$

and since $F$ is continuous, for $h \searrow 0$ the last term $\searrow 0$ which means that

$$
\lim _{h \searrow 0} \frac{X(t) \ominus X(t+h)}{-h}=F(t, X(t)) .
$$

Similarly,

$$
\lim _{h \searrow 0} \frac{X(t-h) \ominus X(t)}{-h}=\lim _{h \searrow 0} \frac{1}{h} \int_{t-h}^{t} F(s, X(s)) d s=F(t, X(t)) .
$$

It follows that $X$ is ((ii)-gH)-differentiable and is a solution of equation (3.11). 


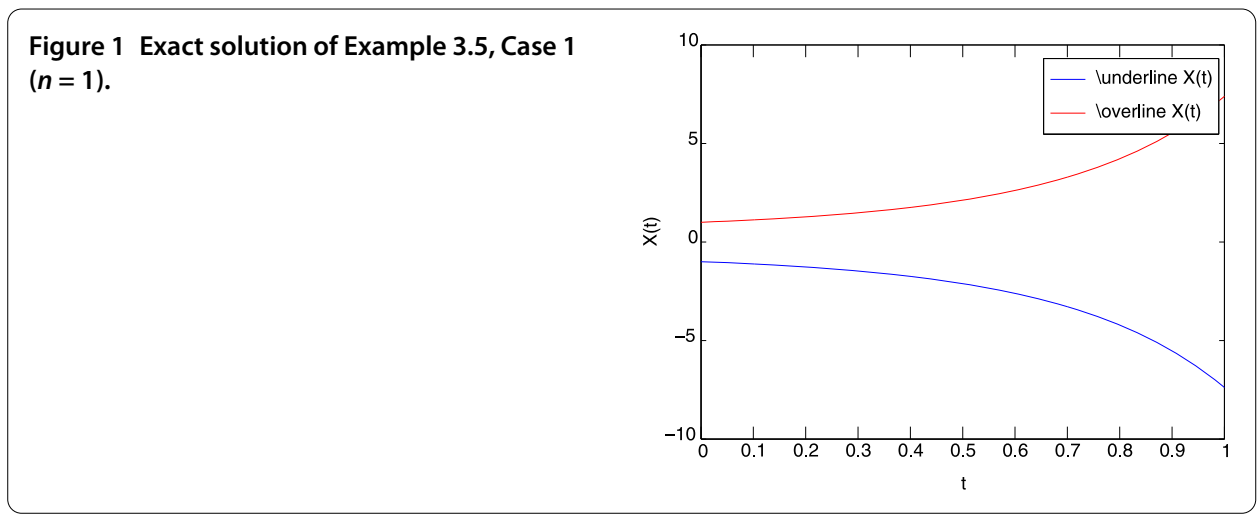

Example 3.5 Consider the box-valued differential equations with initial value:

$$
D_{\mathrm{gH}} X(t)=(1+2 t) X(t), \quad X(0)=[-1,1] \in \mathbb{I}^{1}, \quad t \in[0,1] .
$$

Case 1: $X$ is ((i)-gH)-differentiable, equation (3.15) is equivalent to

$$
\left\{\begin{array}{l}
\underline{X}^{\prime}(t)=(1+2 t) \underline{X}(t), \\
\bar{X}^{\prime}(t)=(1+2 t) \overline{\bar{X}}(t), \\
\underline{X}(0)=-1, \\
\bar{X}(0)=1 .
\end{array}\right.
$$

We get the lower solution $X_{\mathrm{L}}(t)=\left[-e^{t}, e^{t}\right]$ and upper solution $X_{\mathrm{U}}(t)=\left[-e^{3 t}, e^{3 t}\right]$ for the integral equation (3.1) corresponding to problem (3.15), respectively. It is easy to see that all the conditions of Theorem 3.3 are fulfilled and conclusion, there exists a solution for this problem. By solving the above system, we obtain the following exact solution $X(t)=$ $\left[-e^{t(t+1)}, e^{t(t+1)}\right]$. This solution is shown in Figure 1 .

Case 2: $X$ is ((ii)-gH)-differentiable, equation (3.15) is equivalent to

$$
\left\{\begin{array}{l}
\underline{X}^{\prime}(t)=(1+2 t) \bar{X}(t), \\
\bar{X}^{\prime}(t)=(1+2 t) \underline{X}(t), \\
\underline{X}(0)=-1, \\
\bar{X}(0)=1 .
\end{array}\right.
$$

We get the lower solution $X_{\mathrm{L}}(t)=[-\cosh (t)+\sinh (t), \cosh (t)-\sinh (t)]$ and upper solution $X_{\mathrm{U}}(t)=[-\cosh (3 t)+\sinh (3 t), \cosh (3 t)-\sinh (3 t)]$ for the integral equation (3.2) corresponding to problem (3.15), respectively. It is easy to see that all the conditions of Theorem 3.4 are fulfilled and conclusion, there exists a solution for this problem. By solving the above system, we obtain the following exact solution: $X(t)=\left[-\cosh \left(t^{2}+t\right)+\sinh \left(t^{2}+\right.\right.$ $\left.t), \cosh \left(t^{2}+t\right)-\sinh \left(t^{2}+t\right)\right]$. This solution is shown in Figure 2 .

Example 3.6 Consider the box-valued differential equations with initial value:

$$
D_{\mathrm{gH}} X(t)=(1+\sin (t)) X(t), \quad X(0)=\prod_{i=1}^{2}\left[0,1+\frac{1}{i}\right] \in \mathbb{I}^{2}, \quad t \in[0, \pi] .
$$




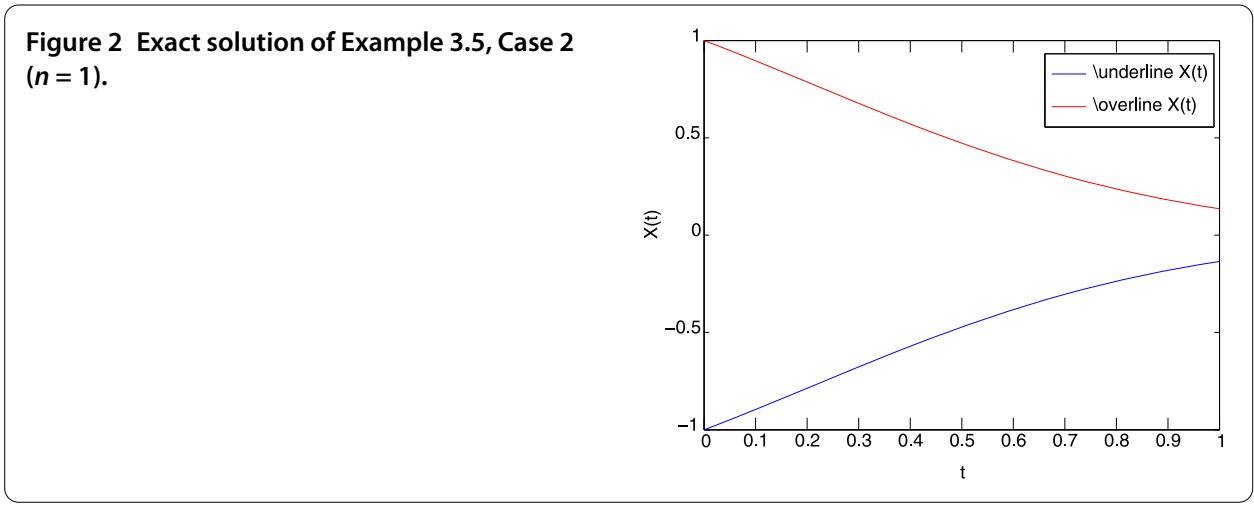

Figure 3 Exact solution of Example 3.6, Case 1 $(n=2)(i=1)$.

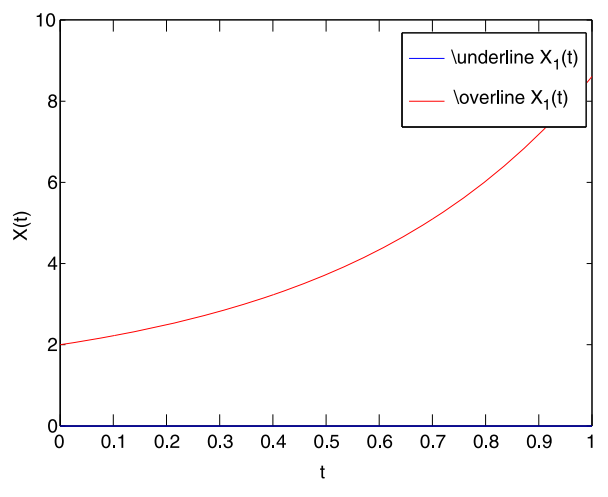

Figure 4 Exact solution of Example 3.6, Case 1 $(n=2)(i=2)$.

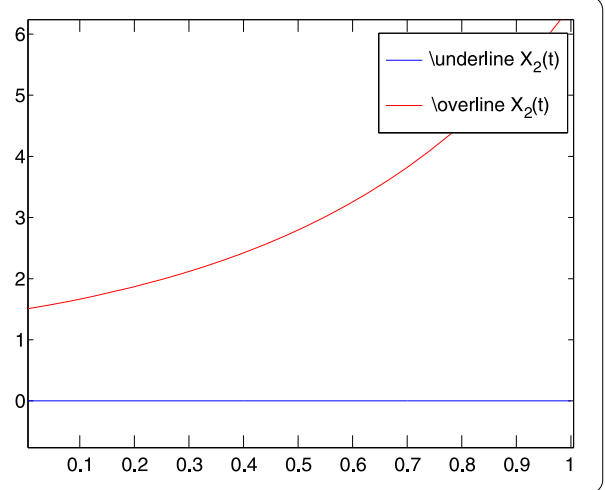

Case 1: $X$ is ((i)-gH)-differentiable, equation (3.18) is equivalent to

$$
\left\{\begin{array}{l}
\underline{X}_{i}^{\prime}(t)=(1+\sin (t)) \underline{X}_{i}(t), \quad i \in\{1,2\}, \\
\bar{X}_{i}^{\prime}(t)=(1+\sin (t)) \bar{X}_{i}(t), \quad i \in\{1,2\}, \\
\underline{X}_{i}(0)=0, \quad i \in\{1,2\}, \\
\bar{X}_{i}(0)=1+\frac{1}{i}, \quad i \in\{1,2\} .
\end{array}\right.
$$

Similarly as in Example 3.5 we get the lower solution $X_{\mathrm{L}}(t)=\prod_{i=1}^{2}\left[0,\left(1+\frac{1}{i}\right) e^{t}\right]$ and upper solution $X_{\mathrm{U}}(t)=\prod_{i=1}^{2}\left[0,\left(1+\frac{1}{i}\right) e^{2 t}\right]$. Moreover, the conditions of Theorem 3.3 are fulfilled and conclusion, there exists a solution for this problem. By solving the above system, we obtain the following exact solution: $X(t)=\prod_{i=1}^{2}\left[0,\left(1+\frac{1}{i}\right) e^{t+1-\cos (t)}\right]$. This solution is shown in Figures 3 and 4. 


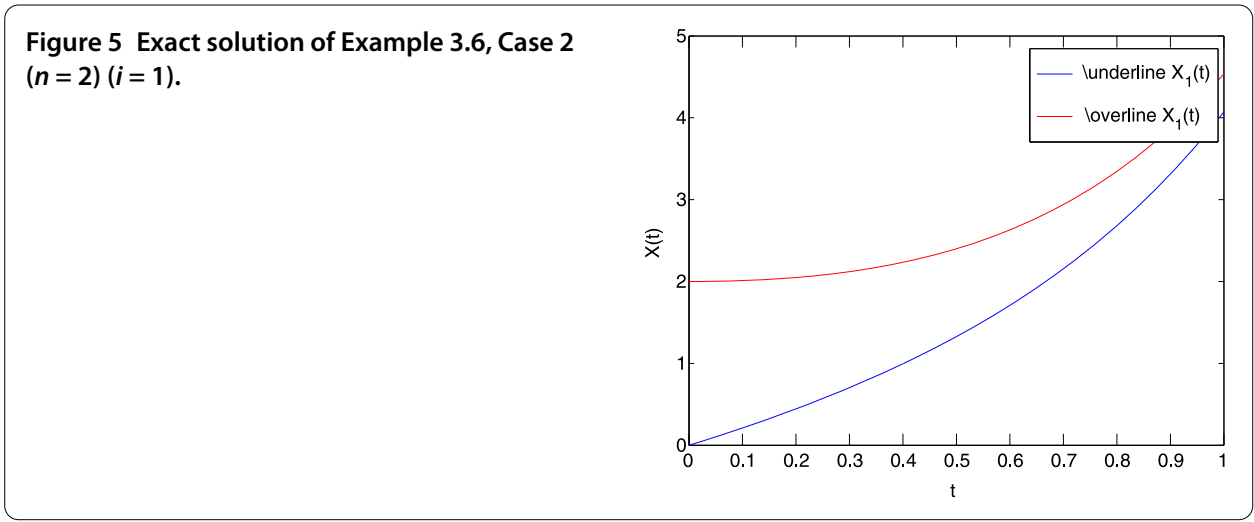

Figure 6 Exact solution of Example 3.6, Case 2 $(n=2)(i=2)$.

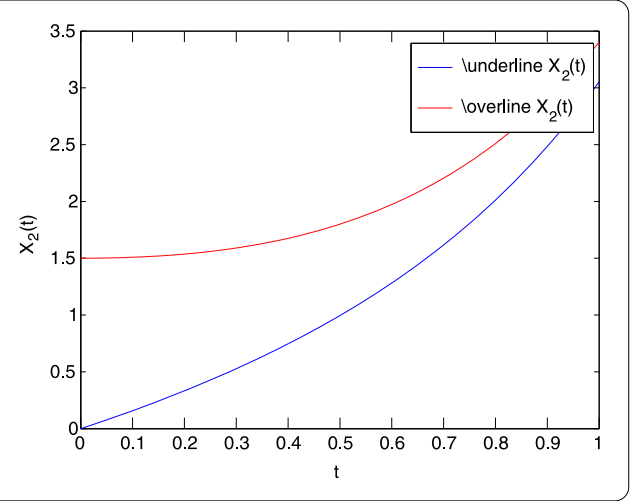

Case 2: $X$ is ((ii)-gH)-differentiable, equation (3.18) is equivalent to

$$
\left\{\begin{array}{l}
\underline{X}_{i}^{\prime}(t)=(1+\sin (t)) \bar{X}_{i}(t), \quad i \in\{1,2\}, \\
\bar{X}_{i}^{\prime}(t)=(1+\sin (t)) \underline{X}_{i}(t), \quad i \in\{1,2\}, \\
\underline{X}_{i}(0)=0, \quad i \in\{1,2\}, \\
\bar{X}_{i}(0)=1+\frac{1}{i}, \quad i \in\{1,2\} .
\end{array}\right.
$$

By solving the above system, we obtain the following exact solution:

$$
X(t)=\prod_{i=1}^{2}\left[\left(1+\frac{1}{i}\right) \sinh (t+1-\cos (t)),\left(1+\frac{1}{i}\right) \cosh (t+1-\cos (t))\right] \text {. }
$$

This solution is shown in Figures 5 and 6.

\section{Conclusion}

Using the method of upper and lower solutions, an existence result of solutions for box integral and differential equations is studied. Also, the monotone iterative technique is developed and the existence results for maximal and minimal solutions are obtained.

\section{Competing interests}

The author declares that they have no competing interests.

\section{Acknowledgements}

The author would like to express gratitude to the anonymous referees for their helpful comments and suggestions, which have greatly improved the paper. 


\section{References}

1. De Blasi, FS, lervolino, F: Equazioni differenziali con soluzioni a valore compatto convesso. Boll. Unione Mat. Ital. 4 , 491-501 (1969)

2. Agarwal, RP, O'Regan, D: Existence for set differential equations via multivalued operator equations. Differ. Equ. Appl. 5, 1-5 (2007)

3. Hoa, NV, Phu, ND: On maximal and minimal solutions for set-valued differential equations with feedback control. Abstr. Appl. Anal. 2012, Article ID 816218 (2012). doi:10.1155/2012/816218

4. Quang, LT, Phu, ND, Hoa, NV, Vu, H: On the maximal and minimal solutions for set integro-differential equations with feedback control. Nonlinear Stud. 20, 39-56 (2013)

5. Bhaskar, TG, Lakshmikantham, V: Set differential equations and flow invariance. J. Appl. Anal. 82, 357-368 (2003)

6. De Blasi, FS, Lakshmikantham, V, Bhaskar, TG: An existence theorem for set differential inclusions in a semilinear metric space. Control Cybern. 36, 571-582 (2007)

7. Lakshmikantham, V, Bhaskar, TG, Devi, JV: Theory of Set Differential Equations in Metric Spaces. Cambridge Scientific Publisher, Cambridge (2006)

8. Malinowski, MT: Second type Hukuhara differentiable solutions to the delay set-valued differential equations. Appl. Math. Comput. 218, 9427-9437 (2012)

9. Malinowski, MT: On set differential equations in Banach spaces - a second type Hukuhara differentiability approach. Appl. Math. Comput. 219(1), 289-305 (2012)

10. Phu, ND, Hoa, NV, Vu, H: On comparisons of set solutions for fuzzy control integro-differential systems. J. Adv. Res. Appl. Math. 4, 84-101 (2012). doi:10.5373/jaram.1081.082011

11. Stefanini, L, Bede, B: Generalized Hukuhara differentiability of interval-valued functions and interval differential equations. Nonlinear Anal. TMA 71, 1311-1328 (2009). doi:10.1016/j.na.2008.12.005

12. Stefanini, L, Bede, B: Some notes on generalized Hukuhara differentiability of interval-valued functions and interval differential equations. Working Paper Series in Economics, Mathematics and Statistics, WP-EMS 2012/8, Univ. Urbino 'Carlo Bo'

13. Malinowski, MT: Interval Cauchy problem with a second type Hukuhara derivative. Inf. Sci. 213, $94-105$ (2012) doi:10.1016/j.ins.2012.05.022

14. Malinowski, MT: Interval differential equations with a second type Hukuhara derivative. Appl. Math. Lett. 24 2118-2123 (2011)

15. An, TV, Hoa, NV, Phu, ND: Global existence of solutions for interval-valued integro-differential equations under generalized H-differentiability. Adv. Differ. Equ. 2013, 217 (2013). doi:10.1186/1687-1847-2013-217

16. An, TV, Phu, ND, Hoa, NV: A note on solutions of interval-valued Volterra integral equations. J. Integral Equ. Appl. 26, 1-14 (2014)

17. Hukuhara, M: Intégration des applications mesurables dont la valeur est un compact convexe. Funkc. Ekvacioj 10 205-229 (1967)

18. Moore, R, Lodwick, W: Interval analysis and fuzzy set theory. Fuzzy Sets Syst. 135, 5-9 (2003)

19. Moore, RE: Methods and Applications of Interval Analysis. Studies in Applied Mathematics. SIAM, Philadelphia (1979)

20. Alefeld, G, Mayer, G: Interval analysis: theory and applications. J. Comput. Appl. Math. 121, 421-464 (2000)

21. Kolev, LV: Interval Methods for Circuit Analysis. World Scientific, Singapore (1993)

22. Lupulescu, V: Hukuhara differentiability of interval-valued functions and interval differential equations on time scales. Inf. Sci. 248, 50-67 (2013)

23. Agarwal, RP, O'Regan, D, Lakshmikantham, V: Viability theory and fuzzy differential equations. Fuzzy Sets Syst. 151 563-580 (2005)

24. Agarwal, RP, O'Regan, D, Lakshmikantham, V: A stacking theorem approach for fuzzy differential equations. Nonlinear Anal. TMA 55, 299-312 (2003)

25. Alikhani, R, Bahrami, F, Jabbari, A: Existence of global solutions to nonlinear fuzzy Volterra integro-differential equations. Nonlinear Anal. TMA 75, 1810-1821 (2012)

26. Alikhani, R, Bahrami, F: Global solutions for nonlinear fuzzy fractional integral and integrodifferential equations. Commun. Nonlinear Sci. Numer. Simul. 18, 2007-2017 (2013)

27. Allahviranloo, T, Khezerloo, M, Sedaghatfar, O, Salahshour, S: Toward the existence and uniqueness of solutions of second-order fuzzy Volterra integro-differential equations with fuzzy kernel. Neural Comput. Appl. 22, S133-S141 (2013)

28. Hajighasemi, S, Allahviranloo, T, Khezerloo, M, Khorasany, M, Salahshour, S: Existence and uniqueness of solutions of fuzzy Volterra integro-differential equations. In: Information Processing and Management of Uncertainty in Knowledge-Based Systems. Applications. Communications in Computer and Information Science, vol. 81, pp. 491-500 (2010)

29. Hoa, NV, Tri, PV, Dao, TT: Some global existence results and stability theorem for fuzzy functional differential equations. J. Intell. Fuzzy Syst. (2013, accepted). doi:10.3233/IFS-141315

30. Hoa, NV, Tri, PV, Phu, ND: Sheaf fuzzy problems for functional differential equations. Adv. Differ. Equ. 2014,156 (2014). doi:10.1186/1687-1847-2014-156

31. Hoa, NV, Phu, ND: Fuzzy functional integro-differential equations under generalized H-differentiability. J. Intell. Fuzzy Syst. 26, 2073-2085 (2014)

32. Bede, B, Gal, SG: Generalizations of the differentiability of fuzzy-number-valued functions with applications to fuzzy differential equations. Fuzzy Sets Syst. 151, 581-599 (2005)

33. Malinowski, MT: Random fuzzy differential equations under generalized Lipschitz condition. Nonlinear Anal., Real World Appl. 13, 860-881 (2012)

34. Malinowski, MT: Existence theorems for solutions to random fuzzy differential equations. Nonlinear Anal. TMA 73, 1515-1532 (2010)

35. Malinowski, MT: On random fuzzy differential equations. Fuzzy Sets Syst. 160, 3152-3165 (2009)

36. $\mathrm{Vu}, \mathrm{H}, \mathrm{Hoa}, \mathrm{NV}, \mathrm{Phu}, \mathrm{ND}$ : The local existence of solutions for random fuzzy integro-differential equations under generalized H-differentiability. J. Intell. Fuzzy Syst. 26, 2701-2717 (2014). doi:10.3233/IFS-130940 
37. Vu, H, Dong, LS, Hoa, NV: Random fuzzy functional integro-differential equations under generalized Hukuhara differentiability. J. Intell. Fuzzy Syst. (2013, accepted). doi:10.3233/IFS-131116

38. Malinowski, MT: Strong solutions to stochastic fuzzy differential equations of Itô type. Math. Comput. Model. 55 , 918-928 (2012)

39. Malinowski, MT: Itô type stochastic fuzzy differential equations with delay. Syst. Control Lett. 61, 692-701 (2012)

40. Malinowski, MT: Some properties of strong solutions to stochastic fuzzy differential equations. Inf. Sci. 252, 62-80 (2013)

41. Malinowski, MT: Approximation schemes for fuzzy stochastic integral equations. Appl. Math. Comput. 219, 11278-11290 (2013)

42. Malinowski, MT: On a new set-valued stochastic integral with respect to semimartingales and its applications. J. Math. Anal. Appl. 408, 669-680 (2013)

43. Akin, Ö, Khaniyev, T, Oruc, Ö, Türksen, IB: An algorithm for the solution of second order fuzzy initial value problems. Expert Syst. Appl. 40, 953-957 (2013)

44. Allahviranloo, T, Amirteimoori, A, Khezerloo, M, Khezerloo, S: A new method for solving fuzzy Volterra integro-differential equations. Aust. J. Basic Appl. Sci. 5, 154-164 (2011)

45. Allahviranloo, T, Salahshour, S, Abbasbandy, S: Solving fuzzy fractional differential equations by fuzzy Laplace transforms. Commun. Nonlinear Sci. Numer. Simul. 17, 1372-1381 (2012). doi:10.1016/j.cnsns.2011.07.005

46. Allahviranloo, T, Abbasbandy, S, Sedaghatfar, O, Darabi, P: A new method for solving fuzzy integro-differential equation under generalized differentiability. Neural Comput. Appl. 21, 191-196 (2012). doi:10.1007/s00521-011-0759-3

47. Allahviranloo, T, Salahshour, S: Euler method for solving hybrid fuzzy differential equation. Soft Comput. 15, 1247-1253 (2011)

48. Salahshour, S, Allahviranloo, T: Applications of fuzzy Laplace transforms. Soft Comput. 17, 145-158 (2013)

49. Salahshour, S, Allahviranloo, T: Application of fuzzy differential transform method for solving fuzzy Volterra integral equations. Appl. Math. Model. 37, 1016-1027 (2013)

50. Bede, B, Rudas, IG, Bencsik, AL: First order linear fuzzy differential equations under generalized differentiability. Inf. Sci. $177,1648-1662(2007)$

51. Hoa, NV, Phu, ND: Global existence of solutions for interval-valued second-order differential equations under generalized Hukuhara derivative. Adv. Differ. Equ. 2013, 290 (2013). doi:10.1186/1687-1847-2013-290

52. Phu, ND, An, TV, Hoa, NV, Hien, TH: Interval-valued functional differential equations under dissipative conditions. Adv. Differ. Equ. 2014, 198 (2014). doi:10.1186/1687-1847-2014-198

53. Hoa, NV, Phu, ND, Tung, TT, Quang, LT: Interval-valued functional integro-differential equations. Adv. Differ. Equ. 2014 177 (2014). doi:10.1186/1687-1847-2014-177

doi:10.1186/1687-1847-2014-223

Cite this article as: Quang: On the solutions for box differential equations under generalized Hukuhara derivative.

Advances in Difference Equations 2014 2014:223.

\section{Submit your manuscript to a SpringerOpen ${ }^{\ominus}$ journal and benefit from:}

- Convenient online submission

Rigorous peer review

- Immediate publication on acceptance

- Open access: articles freely available online

- High visibility within the field

- Retaining the copyright to your article 